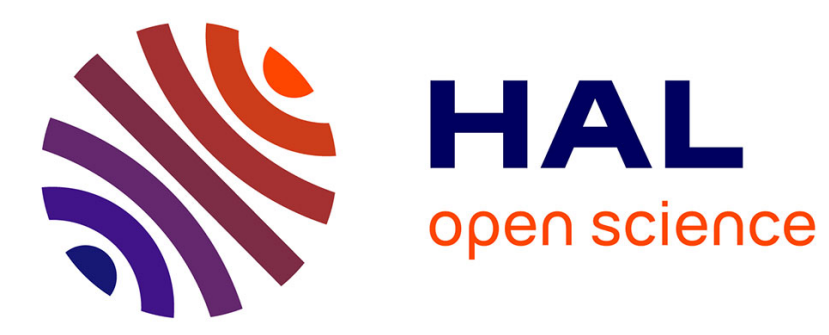

\title{
Point-source imbibition into dry aqueous foams
}

\author{
Rémy Mensire, Jesse T Ault, Elise Lorenceau, Howard A Stone
}

\section{To cite this version:}

Rémy Mensire, Jesse T Ault, Elise Lorenceau, Howard A Stone. Point-source imbibition into dry aqueous foams. EPL - Europhysics Letters, 2016, 113 (4), pp.44002. 10.1209/0295-5075/113/44002 . hal-01345208

\section{HAL Id: hal-01345208 \\ https://hal-enpc.archives-ouvertes.fr/hal-01345208}

Submitted on 10 Oct 2017

HAL is a multi-disciplinary open access archive for the deposit and dissemination of scientific research documents, whether they are published or not. The documents may come from teaching and research institutions in France or abroad, or from public or private research centers.
L'archive ouverte pluridisciplinaire HAL, est destinée au dépôt et à la diffusion de documents scientifiques de niveau recherche, publiés ou non, émanant des établissements d'enseignement et de recherche français ou étrangers, des laboratoires publics ou privés. 


\title{
Point-source imbibition into dry aqueous foams
}

\author{
Rémy Mensire ${ }^{1,2}$, Jesse T. Ault ${ }^{2}$, Elise Lorenceau ${ }^{1}$ and Howard A. Stone ${ }^{2}$ \\ 1 Université Paris-Est, Laboratoire Navier, UMR 8205 CNRS-ENPC-IFSTTAR - 2 allée Kepler F-77420 Champs- \\ sur-Marne, France \\ 2 Department of Mechanical and Aerospace Engineering, Princeton University, Princeton, New Jersey 08544, United \\ States
}

PACS $47.55 . \mathrm{nb}$ - Capillary flows

PACS $82.70 . R r-$ Foams

\begin{abstract}
We use experiments, modeling and numerics to study the imbibition dynamics from a point-source into a homogeneous dry aqueous foam. A distinctive feature of foams compared to solid porous material is that imbibition occurs in the liquid microchannels of the foam called Plateau borders, which have a volume varying in space and time. Dynamics is driven by the capillary pressure and resisted by the viscous and gravity forces in the liquid microchannels. Assuming a constant pressure in the imbibing liquid reservoir, we show that the imbibition front advances and flattens out in time due to gravity, the effect of which is quantified by introducing the Bond number $B$, which compares the gravitational effects to the capillary pressure using the mean bubble radius as the characteristic length. This evolution describes both miscible and immiscible imbibing liquids. For the latter, we introduce the idea of an effective interfacial tension $\gamma_{e f f}$ to take the oil-water interfacial energy into account. The details of the imbibition process are confirmed by experiments and numerics using foams with tangentially immobile interfaces in the channel-dominated model.
\end{abstract}

Introduction. - Aqueous foams have been used extensively in many industrial applications to improve building insulation, to enhance flavours in the food industry, to assist the dismantlement of nuclear power plants, and to improve oil recovery from underground reservoirs [1]. These applications are directly bound to the physical properties of aqueous foams, including their high specific area, low density and non-Newtonian rheology [1-3]. In particular, aqueous foams can be modelled as soft porous media in which imbibition processes can occur.

In this paper, we study the imbibition into a foam from a point source and highlight effects associated with different liquid phases. When the liquid fraction (ratio between the liquid volume and the total volume of the foam) is low, an aqueous foam is a dense assembly of bubbles. The intersections between the bubbles consist of films, vertices and liquid microchannels called Plateau borders, which have a curvature creating a capillary underpressure in the liquid phase. Due to this pressure difference, an aqueous foam has the ability to absorb liquids similar to a sponge. Indeed, the interfacial energy of a wet foam is lower than the interfacial energy of a dry foam since the bubbles are more spherical than in a dry foam. Thus, when a dry aqueous foam is put into contact with the same miscible liquid, imbibition is driven by the reduction of surface energy that occurs when going from a dry to a wetter foam.

Not surprisingly, imbibition phenomena for undeformable and deformable solid porous media have been studied extensively in the literature [4-11]. Also, imbibition of aqueous foams has been studied theoretically and experimentally for different configurations: onedimensional imbibition of the same foaming liquid at atmospheric pressure and pulsed imbibition at constant volumes and $2 \mathrm{D}$ foam drainage at constant flow rate [12-16]. With recent chemical formulations $[17,18]$ making the liquid/air interfaces more rigid, foams absorb not only miscible liquids [12], but also immiscible liquids [19,20], such as organic oils, for the right oil-surfactant combination. This is of considerable interest in oil recovery and soil remediation processes. For those potential applications, oil is generally trapped into micropores at a defined pressure (generally lower than the atmospheric pressure).

We develop a mathematical model and compare with experimental results for the radial imbibition from a point source (at imposed pressure), which mimics liquid extraction from a micropore. Also, we develop a model to ex- 


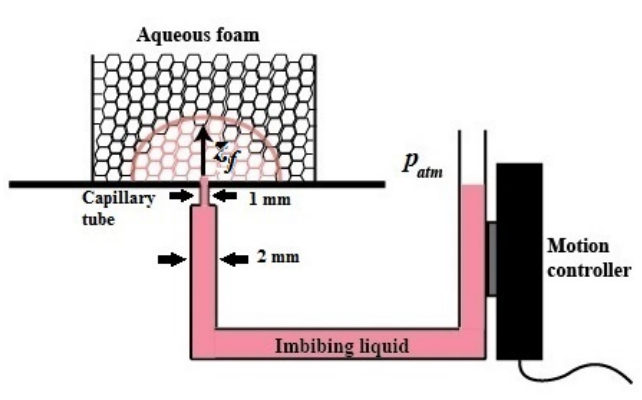

Fig. 1: Experimental set-up. A $30 \mathrm{~cm}$-long PTFE tube $(2 \mathrm{~mm}-$ diameter) is filled with the imbibing liquid (mixtures of foaming solution-glycerol or oil) and terminated by a $1 \mathrm{~cm}$-long capillary tube $(1 \mathrm{~mm}$ diameter $)$ that enables the connection with the foam. A motion controller allows maintainence of the pressure of the liquid phase input at atmospheric pressure $p_{a t m}$.

plain why the imbibition of oil is possible in some cases despite the creation of new oil-water interfaces.

Experiments. - We use a foaming solution of viscosity $\eta=1.4 \mathrm{mPa} . \mathrm{s}$ and density $\rho=1.016 \mathrm{~g} . \mathrm{cm}^{-3}$, based on 0.66 wt. $\%$ of sodium lauryl-dioxyethylene sulfate (SLES; Stepan Co.), 0.34 wt.\% cocoamidopropyl betaine (CAPB; Stepan Co. and Evonik), 0.04 wt.\% myristic acid (MAc; Sigma-Aldrich), and 10 wt.\% glycerol $[17,18]$. As we use different isomers for SLES (because they come from different sources), the air/water surface tension of the foaming solution varies from $\gamma_{o w}=23.7$ to $25.5 \pm 1 \mathrm{mN} / \mathrm{m}$ (measured with the pendant drop method).

As imbibing liquids, we use olive oil and two glycerol solution mixtures, whose wt.\% of glycerol are different; the properties (oil-water surface tension, dynamic viscosity, density) are summarised in Table 1 . We also add a small quantity of fluorescent dyes (1:50) to the imbibing liquid (Yellow Black from Rohm and Haas, Fluorescein from Sigma-Aldrich and Tracerline) to enhance the contrast between the foam and the imbibing liquid. We checked that the addition of dyes does not change the value of $\gamma_{o w}$.

We generate foams with a well-controlled average bubble radius $R_{b}=1-2 \mathrm{~mm}$ by injecting nitrogen or compressed air through a needle into the foaming solution. The rigid interfaces owing to the type of surfactants used yield a stable, monodisperse foam (deviations from the mean bubble radius remain below $5 \%$ ). After generating the foam in a $20 \mathrm{~cm}$-high rectangular column with a 4.5 cm-wide square base, we let it drain and extract at random times at the top of the column a foam sample for which the volume $V$ and the weight $m$ are known. The sample is turned upside down to invert the drainage process, leading to the homogenization of the liquid fraction. The initial liquid fraction of the foam sample is deduced from $\epsilon_{i}=m /(\rho V)$.

For the imbibing reservoir, we use a polycarbonate plate into which a $1 \mathrm{~mm}$-wide hole is drilled. A small capillary tube of $1 \mathrm{~mm}$ diameter is inserted into the hole and slightly displaced upwards into the foam to ensure complete contact between the foam and the reservoir. This capillary

\begin{tabular}{|c|c|c|c|c|c|}
\hline $\begin{array}{c}\text { Imbibing } \\
\text { liquid }\end{array}$ & $\gamma_{\text {ow }}$ & $\eta$ & $\rho$ & $R_{b}$ & $B$ \\
\hline $\begin{array}{c}\text { CAPB+SLES } \\
\text { MAc+80\% gly }\end{array}$ & 0 & 64 & 1.21 & 1 & 0.5 \\
\hline $\begin{array}{c}\text { CAPB+SLES } \\
\text { MAc+65\% gly }\end{array}$ & 0 & 15 & 1.17 & 2 & 1.8 \\
\hline $\begin{array}{c}\text { CAPB+SLES } \\
\text { MAc+10\% gly }\end{array}$ & 0 & 1.4 & 1.016 & 2 & 1.7 \\
\hline Olive oil 1 & 6 & 61 & 0.88 & 1 & 1.5 \\
\hline Olive oil 2 & 6.2 & 68.5 & 0.88 & 2 & 5 \\
\hline Sunflower oil & 4.5 & 55 & 0.91 & 2 & 3 \\
\hline
\end{tabular}

Table 1: Characteristics of the imbibing liquids. $\gamma_{o w}(\mathrm{mN} / \mathrm{m})$ is the oil-water interfacial tension, $\eta$ (mPa.s) the dynamic viscosity, $\rho\left(\mathrm{g} / \mathrm{cm}^{3}\right)$ the density, $R_{b}(\mathrm{~mm})$ the average bubble radius of the foam and $B$ the Bond number defined in (9).

tube is connected to a $20 \mathrm{~cm}$-long and a $2 \mathrm{~mm}$-diameter PTFE tube (Fig. 1). This set of tubes is filled with the imbibing liquids, which completely wet the tubes. The bigger tube is attached to a motion controller that allows the adjustment of the input liquid level at the same height as the output liquid level while the liquid flows through the foam. This system sets a constant atmospheric pressure $p_{a t m}$ at the outlet of the capillary tube. We also use another system without feedback for comparison, by using a $5 \mathrm{~cm}$-wide funnel instead of a motion controller. Indeed, due to the width of the funnel, input pressure remains constant because the output level does not vary much when the liquid is imbibed. Both systems give the same results as shown below.

At time $t=0$, the bottom of the foam touches the point source of the liquid. We record (at 24 frames per second for 3 to 4 minutes) foam imbibition by using fluorescent imaging. Fig. 2 shows a typical image sequence of the imbibition process. The same experiment is repeated for

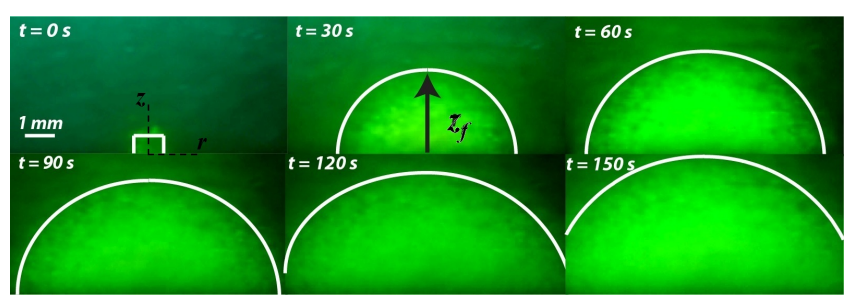

Fig. 2: Snapshots of the imbibition front for a 30-second timescale. The imbibing liquid (glycerol-foaming solution) is fluorescent and the front is defined as the boundary between the brighter and darker fluorescent areas. The liquid-filled tube is displaced upwards by a millimeter in the first snapshot to ensure contact between the source and the foam.

the different imbibing liquids of Table 1 . Thus, different liquids, different viscosities $\eta$, bubble radii $R_{b}$, air-water $\left(\gamma_{a w}\right)$ and oil-water $\left(\gamma_{o w}\right)$ interfacial tensions, and initial liquid fractions $\epsilon_{i}$ are tested. We determine the imbibition front by applying a threshold at the boundary between the brighter and darker fluorescent areas. Using ImageJ soft- 
ware for image processing, we plot the maximum vertical position $z_{f}$ of the front with respect to time.

Results and discussion. - Fig. 3 shows two data sets of for the evolution of the vertical front position $z_{f}(t)$. The experimental data for two different liquids typically have error bars of $\pm 0.5 \mathrm{~mm}$, owing to the determination of the front, especially in the case of miscible liquids, where light diffusion by the films can create a small front width. For both types of liquid, the position of the front moves faster at short times and slows down as time increases. For similar viscosities, oil imbibition is slower than aqueous imbibition.

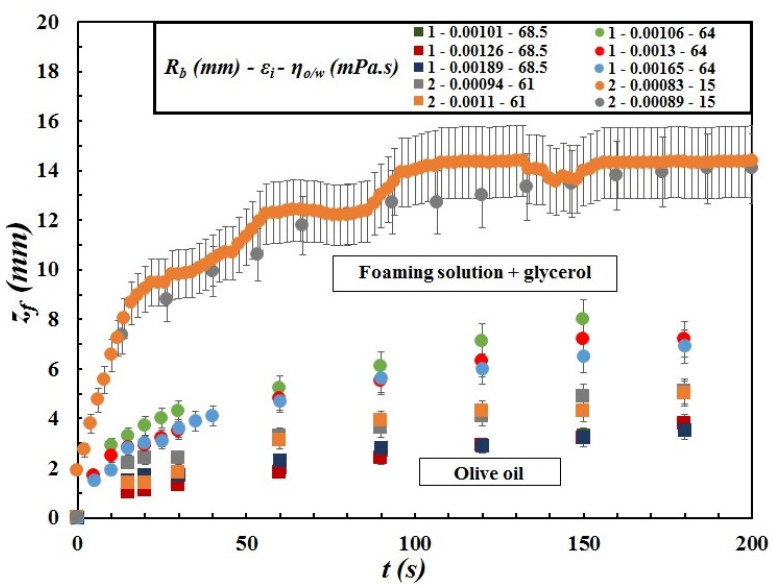

Fig. 3: Evolution of the vertical front position $z_{f}$ with respect to time $t$ for two data sets. The round dots correspond to a glycerolfoaming solution and the square dots to olive oil. The bubble radius $R_{b}$, the initial liquid fraction $\epsilon_{i}$, and the viscosity $\eta_{o} / w$ of the imbibing oil or aqueous solution are indicated, respectively, in the legend.

Miscible liquids. To explain experimental observations, we recall the foam drainage equation $[13,21,22]$ which describes foam imbibition and drainage. We make the following assumptions:

- We consider a dry foam with a constant mean bubble radius $R_{b}$ and a low liquid fraction $\epsilon_{w}<5 \times 10^{-3}$.

- The size of the point source is much smaller than the typical dimensions of the foam and the foam domain can be considered as an infinite space for imbibition.

- We assume isotropy of the foam. We use cylindrical coordinates (radial coordinate $r$ and axial coordinate $z$ ).

Using Darcy's law, we relate the average liquid velocity $\boldsymbol{u}_{\text {Darcy }}$ to the pressure gradient,

$$
\boldsymbol{u}_{\text {Darcy }}=\epsilon_{w} \boldsymbol{u}_{P B}=\frac{k}{\eta_{w}}(-\nabla p+\rho \boldsymbol{g})
$$

where $\boldsymbol{u}_{P B}$ is the mean velocity of a liquid flowing into a Plateau border in a Poiseuille-like flow, $k$ the foam permeability $\left(\mathrm{m}^{2}\right), \epsilon_{w}$ the liquid fraction, $\eta_{w}$ the dynamic viscosity of the invading miscible liquid, $p$ the pressure in the Plateau borders and $\boldsymbol{\nabla}$ depends on $r$ and $z$ only.

To describe the pressure gradient, we resort to a microscopic analysis of the imbibition in the Plateau border.
When a dry aqueous foam is wetted by a miscible liquid, the physical mechanism that enables the imbibition is the reduction of the surface area of the air-water interfaces inside the foam, i.e, the total energy of the system decreases [23]. The liquid flow swells the Plateau borders, creating more surface area. However, if we assume a constant volume of gas in the bubbles, the surface area of the films needs to decrease.

The reduction of interfacial energy is used by the foam to pump a volume $d V$ of liquid at the osmotic pressure $\Pi=p_{a t m}-p$ ( $\Pi$ is identical to the capillary pressure if we assume that the pressure in the bubbles is equal to atmospheric pressure). Thus, we have:

$$
\Pi d V=-\gamma d S \quad, \quad d S<0 \quad,
$$

where $\gamma$ is the interfacial tension, $d S=d S_{f}+d S_{P B}$ is the variation of the air-water area, and $S_{f}$ and $S_{P B}$ are the film and Plateau border area, respectively (Fig. 4a).

Fig. 4: a. Cross-section of a Plateau border for a dry aqueous foam. b. Cross-section of a Plateau border filled with oil for a dry aqueous foam. $S_{P B}$ and $S_{f}$, respectively, denote the areas of the side surfaces of the Plateau borders and the surface of the films that are connected to the Plateau borders. $S_{o}$ represents the area of the oil-water interfaces.

For a dry aqueous and an ordered monodisperse foam, the radius of curvature of a Plateau border $r_{P B}$ is related to the bubble radius $R_{b}$ as $r_{P B}=\delta_{b} R_{b} \epsilon_{w}^{1 / 2}[2,24]$, where $\delta_{b}=1.74$ is a numerical factor deduced from the geometry of a Kelvin cell. As shown analytically in the limit of dry foam in [24], the osmotic pressure can be written as:

$$
\Pi=p_{a t m}-p \approx p_{b}-p \approx \frac{\gamma_{a w}}{r_{P B}} \approx \frac{\gamma_{a w}}{\delta_{b} R_{b} \epsilon_{w}^{1 / 2}}
$$

where $p_{b}$ is the pressure in the bubbles and $\gamma_{a w}$ the airwater interfacial tension. For rigid interfaces, we consider a channel-dominated model for the foam permeability $k$ $[3,25-28]$ :

$$
k=\frac{\delta_{a} \delta_{b}^{2} R_{b}^{2} \epsilon_{w}^{2}}{150}
$$

with $\delta_{a}=\sqrt{3}-\frac{\pi}{2}$, a numerical factor deduced from the cross-sectional area of a Plateau border $A=\delta_{a} r_{P B}^{2}$.

Differential equation for the cross-sectional area of a Plateau border A. By combining (1), (3) and (4) with the unit vector $\boldsymbol{e}_{z}$ directed upwards, we have:

$$
\boldsymbol{u}_{P B}=-\frac{\gamma_{a w} \delta_{a} \delta_{b} R_{b}}{300 \eta_{w} \epsilon_{w}^{1 / 2}} \nabla \epsilon_{w}-\frac{\delta_{a} \delta_{b}^{2} R_{b}^{2} \rho g \epsilon_{w}}{150 \eta_{w}} \boldsymbol{e}_{z}
$$


We consider mass conservation in the Plateau border for the liquid phase, which is expressed as:

$$
\frac{\partial \epsilon_{w}}{\partial t}+\nabla \cdot\left(\epsilon_{w} \boldsymbol{u}_{P B}\right)=0
$$

From (5), (6) and $\epsilon_{w}=A /\left(\delta_{a} \delta_{b}^{2} R_{b}^{2}\right)$, we deduce a nonlinear partial differential equation for the time-space evolution of the cross-sectional area of a Plateau border [13],

$$
\frac{\partial A}{\partial t}=\frac{\gamma_{a w} \delta_{a}^{1 / 2}}{300 \eta_{w}} \nabla \cdot\left(A^{1 / 2} \nabla A\right)+\frac{\rho g}{150 \eta_{w}} \frac{\partial A^{2}}{\partial z}
$$

For the initial condition, we assume that the foam is dry, $\epsilon_{w}=0$. For the boundary conditions, we assume that the liquid fraction and thus the cross-sectional area of a Plateau border far from the point-source is close to 0 . At the point-source, $A=\delta_{a} R_{b}^{2}$, which is the cross-sectional area for the maximum packing of undeformed bubbles. Thus, we have the initial and boundary conditions:

$$
\begin{array}{rlrl}
A(r>0, z>0,0)=0 & , & & A(r, z \rightarrow+\infty, t)=0 \\
A(r \rightarrow+\infty, z, t)=0 & , & A(0,0, t)=\delta_{a} R_{b}^{2}
\end{array}
$$

$\frac{\partial \alpha}{\partial \tau}=B \frac{\partial \alpha^{2}}{\partial Z}+\frac{1}{2}\left(\frac{1}{R} \frac{\partial}{\partial R}\left(R \alpha^{1 / 2} \frac{\partial \alpha}{\partial R}\right)+\frac{\partial}{\partial Z}\left(\alpha^{1 / 2} \frac{\partial \alpha}{\partial Z}\right)\right)$

with the initial and boundary conditions:

$$
\begin{aligned}
& \alpha(R>0, Z>0,0)=0 \quad, \quad \alpha(R, Z \rightarrow+\infty, \tau)=0 \\
& \alpha(R \rightarrow+\infty, Z, \tau)=0 \quad, \quad \alpha(0,0, \tau)=1
\end{aligned}
$$
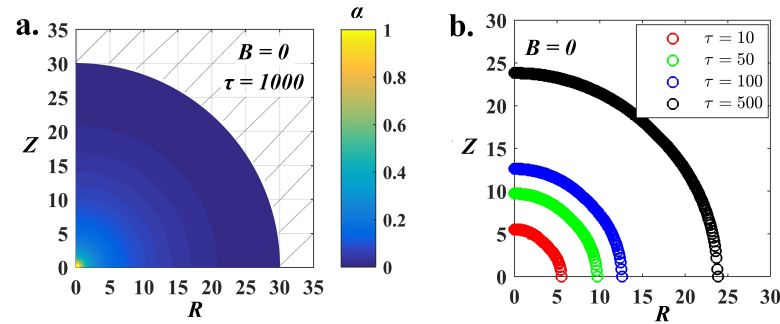

c.
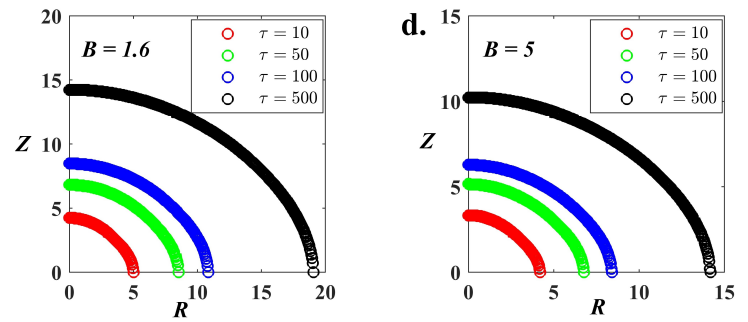

Fig. 5: a. Typical numerical solution of (9) for the dimensionless Plateau border area $\alpha$ for $B=0$ and $\tau=1000$. b-d. Numerical solutions of the imbibition front for different times $\tau=10,50,100$ and 500 with Bond numbers $B=0,1.6$ and 5 .

new oil-water interfaces are also created, which are energetically costly. Therefore, we develop another model to add the influence of these oil-water interfaces.

Rewriting the osmotic pressure from (2), by using the interfaces $d S_{w}$ and $d S_{o}$ for the variations of the air-water and oil-water interfaces, respectively, yields:

$$
\begin{gathered}
\Pi d V=-\left(\gamma_{a w} d S_{w}+\gamma_{o w} d S_{o}\right) \\
\text { with } d S_{w}<0 \quad \text { and } \quad d S_{o}>0
\end{gathered}
$$

Equation (11) can also be expressed as:

$$
\Pi d V=-\gamma_{a w} d S_{w}\left(1+\frac{\gamma_{o w}}{\gamma_{a w}} \frac{d S_{o}}{d S_{w}}\right)=-\gamma_{e f f} d S_{w}
$$

with $\gamma_{e f f}$ an effective interfacial tension:

$$
\gamma_{e f f}=\gamma_{a w}\left(1+\frac{\gamma_{o w}}{\gamma_{a w}} \frac{d S_{o}}{d S_{w}}\right)
$$

For miscible liquids, $\gamma_{o w}=0$, so $\gamma_{e f f}=\gamma_{a w}$ and the osmotic pressure is related to the curvature of the Plateau borders by (3). However, if $\gamma_{o w} \neq 0$, then $\gamma_{\text {eff }}$ accounts for the oil-water interfacial tension and the creation of oil-water interfaces. The osmotic pressure becomes $\Pi=\gamma_{e f f} / r_{P B} \approx \gamma_{e f f} /\left(\delta_{b} R_{b} \epsilon^{1 / 2}\right)$ where $\epsilon$ is the combined liquid fraction for oil and water. Since $d S_{w}<0$, then $\gamma_{e f f}<\gamma_{a w}$. With immiscible liquids, the osmotic pressure is reduced compared to miscible liquids, i.e., the driving force for imbibition is weaker. The value of $\gamma_{e f f}$ can be estimated. In particular, the variation of the airwater interfaces $d S_{w}$ can be decomposed into two contributions, one from the films $d S_{f}$, and the other from the Plateau borders $d S_{P B}$ (Fig. 4b).

Pitois et al. [29] and Hilgenfeldt et al. [30] provide estimates of the film and Plateau border surface areas with 
regard to the liquid fraction in the foam,

$$
\begin{gathered}
S_{f} \approx \frac{3.3}{R_{b}} V_{\text {foam }}(1-\epsilon)\left(1-1.52 \epsilon^{1 / 2}\right)^{2} \\
S_{P B} \approx \frac{V_{\text {foam }}}{1.5 R_{b}}\left(\frac{\pi}{\sqrt{3} \delta_{a}}\right) \epsilon^{1 / 2}
\end{gathered}
$$

where $V_{\text {foam }}$ is a volume of foam. Differentiating (14a) and (14b) with respect to $\epsilon$ in the dry limit yields,

$$
\begin{aligned}
& d S_{f} \approx-\frac{V_{\text {foam }}}{R_{b}} \frac{(3.3)(3.04)}{2 \epsilon^{1 / 2}} d \epsilon \\
& d S_{P B} \approx \frac{V_{\text {foam }}}{R_{b}} \frac{\pi}{3 \sqrt{3} \delta_{a} \epsilon^{1 / 2}} d \epsilon
\end{aligned}
$$

In the dry limit, we can assume as a first approximation that $d S_{o} \approx d S_{P B}$ and neglect the oil-water interface at the top of the oil slug (Fig. 4b). This assumption is in agreement with recent Surface Evolver simulations of the shape of an oil slug in a single Plateau border [27]. In our case, the ratio $\gamma_{o w} / \gamma_{a w} \approx 0.2$ is low and the ratio between the equivalent spherical radius for an oil slug embedded within the Plateau border and the radius of curvature of the Plateau border is close to 1 due to the very low liquid fraction. Thus, by using (15a) and (15b), we have:

$$
\gamma_{e f f} \approx \gamma_{a w}+\gamma_{o w} \frac{d S_{P B}}{d S_{f}+d S_{P B}} \approx \gamma_{a w}\left(1-3 \frac{\gamma_{o w}}{\gamma_{a w}}\right)
$$

With this effective interfacial tension, the osmotic pressure is reduced by a factor that depends on the ratio between the oil-water and the air-water interfacial tensions. More precisely, the governing equation for $A$ is the same as developed previously for aqueous liquids in (7) with $A$, the cross-sectional area of the Plateau border for both oil and water phases, $\gamma_{e f f}$, instead of $\gamma_{a w}$, and $\eta_{o}$ the oil viscosity:

$$
\frac{\partial A}{\partial t}=\frac{\gamma_{e f f} \delta_{a}^{1 / 2}}{300 \eta_{o}} \nabla \cdot\left(A^{1 / 2} \nabla A\right)+\frac{\rho g}{150 \eta_{o}} \frac{\partial A^{2}}{\partial z}
$$

We only use $\eta_{o}$ because the viscous dissipation occurs predominantly in the oil phase as shown by Piroird et al. [19] in the limit of $\eta_{o} / \eta_{w} \gg 1$. Eq. (17) is identical to (7) and can be solved numerically, except with a smaller air-water interfacial tension. The smaller capillary pressure induced by the oil phase is in agreement with our experiments: for the same elapsed time, the vertical front position of the oil is smaller than the aqueous case, as shown in Fig. 3.

In Fig. 6 , we rescale $z_{f}$ by $R_{b}$ and $t$ by the capillary time $\left(150 / \delta_{a}\right) \eta_{o / w} R_{b} / \gamma_{e f f}$, which is the same scaling as in (9) written with $\gamma_{\text {eff }}$ instead of $\gamma_{a w}$, and the viscosity of oil or aqueous solutions $\eta_{o} w$. The log-log plot in the dimensionless variables displays a reasonable collapse between the experimental data and the numerical solution for all miscible and immiscible imbibing liquids for the range of non-zero Bond numbers between 0.5 and 5 . The collapse occurs at shorter $\tau$ for immiscible liquids, due to the smaller scaling in time induced by $\gamma_{e f f}$.

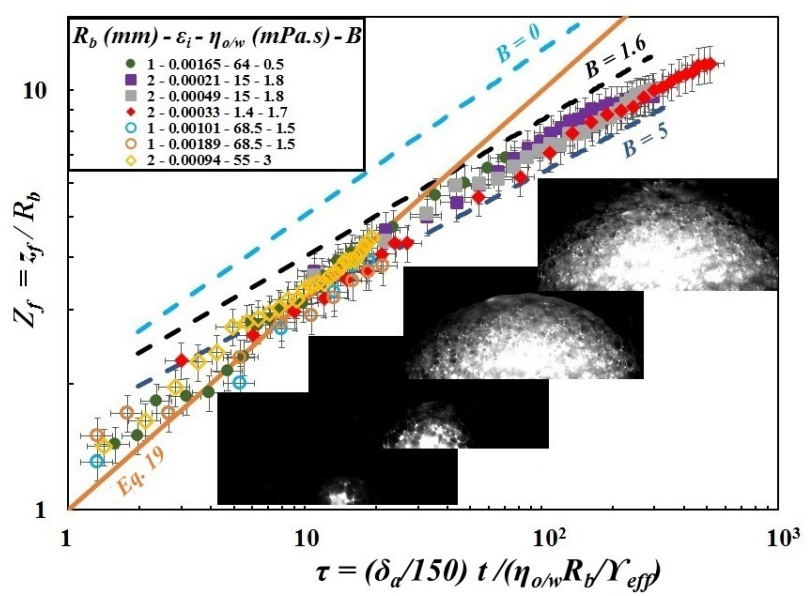

Fig. 6: Vertical front position $z_{f}$ with respect to time in dimensionless coordinates for immiscible oils (open dots) and miscible aqueous liquids (closed dots). The experimental curves are obtained for two $R_{b}=1-2 \mathrm{~mm}$, different $\epsilon_{i}, \eta_{o} / w$ and $B$. The numerical solutions of (9) for Bond numbers $B=0,1.6$ and 5 , deduced from (9), are plotted by the dashed lines. The self-similar power law evolution in $\tau^{1 / 2}$ in the no-gravity case (Eq. 19) is shown.

In both miscible and immiscible cases, the numerical solutions that include the capillary pressure gradient and gravity are comparable to the experimental data. Gravity should be considered, since the numerical solution with $B=0$ largely deviates from the experimental data, and gravitational effects flatten out the imbibition profiles very quickly as shown in Fig. 5. However, our model deviates from the data at short times. Indeed when the front position is of the order of magnitude of one bubble size $\left(R^{*}<2\right)$, the Darcy model for the average velocity is not adequate, as imbibition occurs in individual Plateau borders. Also, $\epsilon \neq 0$ around the point source.

Analytical solution with $B=0$. An analytical scaling for the front position in the no-gravity case $(B=0)$, which is encountered in microgravity conditions, can be found by assuming spherical symmetry with the dimensionless spherical distance $\tilde{R}$. We introduce the dimensionless self-similar variable $\zeta=\tilde{R} / \tau^{1 / 2}$. Substituting $\zeta$ into (9), written with $\gamma_{e f f}$ and $\eta_{o / w}$, we find that $\alpha$ is the solution of the ordinary differential equation:

$$
\zeta^{3} \frac{d \alpha}{d \zeta}+\frac{d}{d \zeta}\left(\zeta^{2} \alpha^{1 / 2} \frac{d \alpha}{d \zeta}\right)=0
$$

The first boundary condition is $\alpha\left(\zeta_{f}\right)=0$, where $\zeta_{f}=$ $\left(\frac{150 \eta_{o} / w}{\gamma_{e f f} \delta_{a} R_{b}}\right)^{1 / 2} \frac{r_{f}}{t^{1 / 2}}$ is a constant which then yields the spherical front radius $r_{f}(t)$. A local analysis at the front provides a second boundary condition and uniquely determines the solution.

Setting $\zeta_{f}^{4} \alpha^{*}=\alpha$ yields the same equation as (18) except with $\zeta_{f}=1$. Therefore, we can take $\zeta_{f}=1$ without loss of generality. The wetting front $r_{f}$ is given by:

$$
\frac{r_{f}(t)}{R_{b}}=\left(\frac{\delta_{a} \gamma_{e f f}}{150 \eta_{o / w} R_{b}} t\right)^{1 / 2}
$$


This solution is plotted in Fig. 6. The $t^{1 / 2}$ power law result recalls the $1 \mathrm{D}$ diffusive imbibition in a Hele-Shaw cell observed in $[12,31]$. However, the collapse between the PDE solution with $B=0$ and the self-similar solution in $t^{1 / 2}$ occurs at long times for $\tau>10^{4}$. The value of $\alpha$ at the point source is equal to 1 . Yet, the self-similar solution blows up and is not consistent around the point source [32]. Also, given the values of the Bond numbers from 0.5 to 5 , the diffusive solution (and thus the no-gravity approximation) is not valid over our experimental range.

Conclusions. The radial imbibition from a point source for all types of liquid into a dry aqueous foam has been studied theoretically, numerically and experimentally. Theory, numerics and experiments are in good agreement. The results demonstrate that gravitational effects are comparable to the capillary pressure. Our result differs from the $t^{1 / 3}$ power law developed by Xiao et al. [33] for radial imbibition in undeformable porous media with a constant permeability $k$. We account for the deformability of the Plateau borders (time variations and non-constant $k$ ), which produces modifications to the power law. We assume that viscous dissipation occurs in the Plateau borders and adopt a channel-dominated model for the liquid flow through the foam. Also, we consider gravity effects, which are neglected in [33], since $B \ll 1$ due to the micron size of the glass beads in their porous matrix.

From our results, we identify two criteria that define the imbibition efficiency. The first is the ratio between the oil-water and the air-water interfacial tension that predicts the imbibition strength. The second one is the Bond number $B$. The lower $B$, the less the effect of gravity and the more liquid is imbibed. Decreasing the bubble radius is the main option for reducing $B$ (apart from microgravity). In conclusion, our system mimics the ability of a foam to remove liquids from a point source, such as a pore or a fracture, and complements the wetting theories in foam engineering.

We thank I. Cantat and O. Pitois for advice on the experimental process and the theoretical analysis. We acknowledge H. Kim and J. Wexler for their help on interfacial tension and viscosity measurements. This work has benefitted from the financial support of the Agence Nationale de la Recherche (ANR-11-JS09-012-Wolf), the Department of Mechanical and Aerospace Engineering at Princeton University, and Paris-Est University.

\section{REFERENCES}

[1] P. Stevenson, Foam Engineering: Fundamentals and Applications (Wiley) 2012.

[2] D. Weaire and S. Hutzler, The Physics of Foams (Oxford Univ. Press) 1999.

[3] I. Cantat, S. Cohen-Addad, F. Elias, F. Graner, R. Hohler, O. Pitois, F. Rouyer and A. Saint-Jalmes,
Foams: Structure and Dynamics (Oxford Univ. Press) ${ }_{28}^{28}$ 2013.

[4] R. LucAs, Kolloid Z., 23 (1918) 15.

[5] E. W. Washburn, Phys. Rev. Lett., 17 (1921) 273.

[6] P. G. Gennes, F. Brochard-Wyart and D. Quere, Capillarity and Wetting Phenomena: Drops, Bubbles, Pearls and Waves (Springer) 2004.

[7] M. Reyssat, L. Courbin, E. Reyssat and H. A. Stone, J. Fluid. Mech., 615 (2008) 335-344.

[8] A. Ponomarenko, D. Quere and C. Clanet, J. Fluid. Mech., 666 (2011) 146.

[9] L. TANG and Y. TANG, J. Phys. II, 4 (1994) 881.

[10] S. Mendez, E. M. Fenton, G. R. Gallegos, D. N Petsev, S. S. Sibbett, H. A. Stone, Y. Zhang, and G. P. LOPEZ, Langmuir, 26 (2009) 1380-1385.

[11] C. Duprat, J. M. Aristoff and H. A. Stone, J. Fluid. Mech., 679 (2011) 641.

[12] H. Caps, S. Cox, H. Decaumer, D. Weaire and N VANDEWAlle, Colloids and Surfaces A, 261 (2005) 131.

[13] S. Koehler, H. A. Stone, M. Brenner and J. Eggers, Phys. Rev. E, 58 (1998) 2097.

[14] S. Koehler, S. Hilgenfeldt and H. A. Stone, J. Coll. Int. Sci., 276 (2004) 420.

[15] S. J. Cox, D. Weaire and G. Verbist, Eur. Phys. J. B., 40 (2004) 119-121.

[16] S. Hutzler, S. J. Cox and G. Wang, Colloids and Surfaces A: Physicochem. Eng. Aspects, 263 (2005) 178.

[17] E. Basheva, D. Ganshev, N. D. Denkov, K. Kasuga, N. SATOH and K.TsujII, Langmuir, 16 (2000) 1000.

[18] K. Golemanov, N. D. Denkov, S. Tcholakova, M. Vethamuthu and A. Lips, Langmuir, 24 (2008) 99569961.

[19] K. Piroird and E. Lorenceau, Phys. Rev. Lett., 111 (2013) 234503.

[20] R. Mensire, K. Piroird and E. Lorenceau, Phys. Rev. E, 92 (2015) 053014.

[21] G. Verbist and D. Weaire, Europhys. Lett., 26 (1994) 631.

[22] G. Verbist, D. Weaire and A. M. Kraynik, J. Phys. Condens., 8 (1996) 3715.

[23] R. Hohler, Y. Y. Cheung Sang, E. Lorenceau and S. COHEN-ADDAD, Langmuir, 24 (2008) 418-425.

[24] N. Kern and D. Weaire, Philo. Mag., 83 (2003) 2973.

[25] S. Koehler, S. Hilgenfeldt and H. A. Stone, Langmuir, 16 (2000) 6327.

[26] S. Cohen-Addad, R. Hohler and O. Pitois, Ann. Rev. Fluid. Mech., 45 (2013) 241.

[27] S. J. Neethling, G. Morris and P. R. Garrett, Langmuir, 27 (2011) 9738-9747.

[28] A. Nguyen, J. Coll. Int. Sci., 249 (2002) 194.

[29] O. Pitois, E. Lorenceau, N. Louvet and F. Rouyer, Langmuir, 25 (2009) 97-100.

[30] S. Hilgenfeldt, S. Koehler and H. A. Stone, Phys. Rev. Lett., 86 (2001) 4704.

[31] A. Saint-Jalmes, S. Marze, H. Ritacco, D. Langevin, S. Bail, J. Dubail, L. Guingot, G. Roux, P. Sung and L. Tosini, Phys. Rev. Lett., 98 (2007) 058303.

[32] I. Christov and H. A. Stone, Proc. Natl. Acad. Sci., 10 (2012) 1073

[33] J. Xiao, H. A. Stone and D. Attinger, Langmuir, 28 (2012) 4208-4212. 